\title{
Distribución de Phytophthora cactorum en el perfil de un suelo cultivado con frutilla (Fragaria x ananassa).
}

\author{
María Josefina Iribarren ${ }^{1}$, Beatriz Angela González², Susana Filippini ${ }^{3}$
}

\begin{abstract}
${ }^{1}$ Elementos de Diagnóstico en Recursos Naturales, Departamento de Tecnología. ${ }^{2}$ Fitopatología, Departamento de Tecnología. ${ }^{3}$ Estadística, Departamento de Ciencias Básicas. Universidad Nacional de Luján, UNLU. Ruta 5 y 7, Luján (6700). Buenos Aires, Argentina.

Autor para correspondência: María Josefina Iribarren (miribarren@unlu.edu.ar)

Data de chegada: 31/08/2009. Aceito para publicação em: 02/01/2012.
\end{abstract}

\section{RESUMEN}

Iribarren, M.J.; González, B.A.; Filippini, S. Distribución de Phytophthora cactorum en el perfil de un suelo cultivado con frutilla (Fragaria x ananassa). Summa Phytopathologica, v.38, n.1, p.17-23, 2012.

Phytophthora cactorum es el agente causal de la podredumbre seca que produce importantes pérdidas en los cultivos de frutilla (Fragaria x ananassa). Este patógeno persiste como oosporas formadas principalmente en los frutos afectados, los que quedan en la superficie del suelo. En un lote representativo de los cultivos de frutillas de la zona noreste de la provincia de Buenos Aires, Argentina, y después de haberse levantado la plantación y labrado el suelo, se buscó establecer la distribución espacial de las oosporas de $P$. cactorum remanentes. El muestreo se realizó en tres bloques ubicados a lo largo de una transecta diagonal al terreno y a dos profundidades en el perfil del suelo. También se caracterizó la topografía del terreno y parámetros físicos del suelo de interés.
La detección de $P$. cactorum se realizó mediante el empleo de cebos. No se encontraron diferencias significativas en cuanto a la presencia del patógeno en los distintos puntos muestreados sobre el terreno, a la escala de análisis. Sólo se observó una pequeña tendencia a la disminución del inóculo en profundidad. El número de recuentos positivos en las profundidades $\mathrm{A}$ y $\mathrm{AB} / \mathrm{BA}$ fueron respectivamente 30 y 22 en un total de 72 muestras. Tampoco hubo diferencias significativas en densidad aparente, humedad equivalente y macroporosidad del suelo. Si bien la distribución en superficie de $P$. cactorum pudo deberse fundamentalmente a procesos naturales, la labranza del suelo posibilitó la dispersión de oosporas viables en profundidad.

Palabras clave adicionales: dispersión espacial, podredumbre seca, Fragaria x ananassa.

\section{RESUMO}

Iribarren, M.J.; González, B.A.; Filippini, S. Distribuição de Phytophthora cactorum no perfil de um solo cultivado com morango (Fragaria x ananassa). Summa Phytopathologica, v.38, n.1, p.17-23, 2012.

Phytophthora cactorum é o agente causal da podridão seca, que produz importantes perdas nos cultivos de morango (Fragaria $\mathrm{x}$ ananassa). Este patógeno sobrevive no solo como oósporos, formados a partir de frutos afetados, os quais permanessem na superfície do solo. Assim, depois que a plantação foi removida e o solo gradeado boscou-se establecer a distribuição espacial dos oósporos de $P$. cactorum remanescentes numa área de cultivo de morango em Buenos Aires, Argentina. A amostragem foi realizada em três blocos localizados ao longo de um transecto diagonal ao terreno e a duas profundidades. Também foram caracterizadas a topografia do terreno e os parâmetros físicos do solo. A detecção de $P$. cactorum foi realizada mediante o emprego do método da isca usando cotiledones de maçã. A escala de análise mostrou que não foi encontrada diferenças significativas em relação a presença de patógenos nos distintos pontos usados como amostra sobre o terreno e somente observou-se uma pequena tendência a diminuição do inóculo na profundidade. O número de amostras positivas nas profundidades $\mathrm{A}$ y $\mathrm{AB} / \mathrm{BA}$ foram respectivamente 30 e 22 um total 72 amostras. Tampouco houve diferenças significativas na densidade aparente, na umidade equivalente e na macroporosidade do solo. A distribuição de $P$. cactorum na superfície pode ter sido decorrente fundamentalmente dos processos naturais, sendo que o preparo do solo pode ter possibilitado a dispersão de oósporos viáveis em profundidade.

Palavras chaves adicionais: distribuição espacial, podridão seca, Fragaria x ananassa.

\section{ABSTRACT}

Iribarren, M.J.; González, B.A.; Filippini, S. Phytophthora cactorum distribution in a soil profile cultivated with strawberry (Fragaria $\mathrm{x}$ ananassa). Summa Phytopathologica, v.38, n.1, p.17-23, 2012.

Phytophthora cactorum is the causal agent of leather rot, which has led to significant losses in strawberry (Fragaria x ananassa) crops. This pathogen persists as oospores mainly formed in infected fruits, which remain in the soil surface. The study was carried out to investigate, after plantation removal and soil tillage, the spatial distribution of the remaining $P$. cactorum oospores in a representative 
lot for strawberry cultivation in the northeast region of the Buenos Aires province, Argentina. Sampling was performed in three blocks located along a transect diagonal to the terrain and at two depth levels in the soil profile. The topography and the soil physical parameters of interest were also evaluated. P. cactorum was detected by using baits. Based on the analysis scale, there were no significant differences regarding pathogen presence at the distinct points set as samples on the soil surface; a slight tendency toward a decrease in the inoculums presence according to depth was observed. The number of positive samples in both depth, A and AB/ $\mathrm{BA}$, was 30 and 22 respectably in 72 samples in total. In addition, there were no significant differences in the soil apparent density, equivalent moisture and macroporosity. P. cactorum distribution on the soil surface may have been fundamentally due to natural mechanisms and soil tillage allowed the spread of viable oospores with depth.

Additional keywords: spatial distribution, leather rot, Fragaria x ananassa.

La frutilla (Fragaria x ananassa) es un cultivo de creciente importancia en Argentina, con una producción de 35.000 toneladas anuales. El mayor incremento en la superficie plantada se ha producido en la región noreste de la provincia de Buenos Aires (30), en la que típicamente se rota con cultivos hortícolas.

Dicha zona posee relieve llano y suelos Argiudoles típicos o vérticos (28) y el clima es templado húmedo (29). Estas características constituyen un ambiente conductivo para $P$. cactorum (1), un importante patógeno de las frutillas en la mayoría de las regiones productoras del mundo (7).

$P$. cactorum es una especie homotalica, por lo que produce oosporas. Estas son las estructuras fundamentales de persistencia de esta especie, pudiendo perdurar libres en el suelo por más de un año (26). Forma raramente clamidosporas y tanto el micelio como los zoosporangios, zoosporas y quistes poseen corta vida, principalmente cuando están sometidos a condiciones ambientales extremas o quedan incluidos en el suelo.

$P$. cactorum posee aislamientos con un elevado nivel de especificidad para algunos hospedantes; tal es el caso de la frutilla $(12,15)$, en la que produce la podredumbre seca de los frutos, así como la podredumbre de corona. El primer síntoma es el que se expresa con mayor frecuencia en América, a diferencia de lo que ocurre en Europa $(6,7)$.

La elevada especificidad de $P$. cactorum hace que la fuente de inóculo primario sólo tenga dos orígenes posibles: uno es la introducción anual en los cultivos de plantines, los que si bien son asintomáticos, pueden tener presencia del pseudohongo (17), muchas veces en el suelo adherido a sus raíces $(9,25)$. La otra fuente de inóculo primario son las oosporas remanentes en el suelo (10).

Estas estructuras se forman mayoritariamente en las frutillas enfermas o momificadas; cuando estos frutos se descomponen y las oosporas quedan libres sobre el suelo sus poblaciones disminuyen porque son afectadas por las elevadas temperaturas del verano (8) y además son inducidas a germinar al quedar expuestas a la luz (2).

En el perfil del suelo las especies de Phytophthora se mueven mediante las zoosporas, cuyo movimiento helicoidal puede ocurrir en los macroporos, pero se encuentra efectivamente asegurado en discontinuidades de gran diámetro como las galerías dejadas por los insectos, las que quedan al desintegrarse las raíces y las grietas estructurales del suelo $(4,5)$.

Las oosporas, al ser inmóviles, sólo pueden ser diseminadas naturalmente por algunos animales presentes en el suelo, por lo que en suelos no roturados se distribuyen superficialmente en el perfil del suelo. Horner \& Wilcox (13), en un estudio realizado en Nueva York en montes de manzanos, encontraron una marcada disminución en las poblaciones de $P$. cactorum con el incremento de la profundidad, con un recuento nulo o una proporción muy baja de oosporas a $20 \mathrm{~cm}$ de profundidad. No hubo asociación entre la presencia de raíces de manzanos colonizadas por el patógeno y la presencia de oosporas en el suelo y sus poblaciones fueron mayores en las zonas más húmedas del terreno.

En el caso de cultivos anuales, el laboreo del suelo produce una distribución vertical de Phytophthora, al incorporar los residuos colonizados; sin embargo la magnitud de este fenómeno puede ser no significativa según lo demostraron Workneh et al. (27) en un estudio en el que comprobaron que el laboreo convencional no incrementó la presencia de $P$. sojae en profundidad con respecto a la plantación directa. Atribuyeron ese efecto a la rápida descomposición de los residuos que resultaron incorporados por el laboreo. Ese efecto puede ser mucho más marcado en el caso de las frutillas, porque la composición de los tejidos de estos frutos hace que su descomposición sea muy rápida. Al mismo tiempo, algunas condiciones de los suelos presentes en la región pueden afectar negativamente la supervivencia de las oosporas de P. cactorum; como la elevada capacidad de retención hídrica, típica de los mismos, que incrementa la actividad de la microflora antagonista.

La distribución de $P$. cactorum en el perfil del suelo afecta directamente la efectividad de los tratamientos que se puedan aplicar para eliminar el inóculo remanente; de allí la necesidad de conocer las características de este fenómeno para poder realizar adecuadas recomendaciones de control.

El objetivo de este trabajo fue estudiar la distribución espacial de las oosporas de $P$. cactorum remanentes en el suelo, una vez que se retiró el cultivo de frutilla, en una situación de producción comercial.

\section{MATERIALES Y MÉTODOS}

Se tomaron muestras de un lote ubicado en Luján, provincia de Bs. As. [34³4'25.57' S; 5908'07.37'O] el que correspondió a un suelo Argiudol típico, caracterizado por la presencia de un horizonte A, seguido por un horizonte $\mathrm{AB} / \mathrm{BA}$ y un horizonte argílico. El mismo al momento del muestreo, se encontraba implantado con un cultivo de frutillas, en el que se constató la presencia de frutos afectados por $P$. cactorum. Al momento del muestreo el suelo se hallaba labrado, los restos de plantas de frutillas se habían desintegrado totalmente y se encontraba preparado en camellones, listo para la implantación del siguiente cultivo.

El lugar de muestreo se ubicó en una zona central del lote, en una parcela de $12 \mathrm{~m}$ de ancho por $35 \mathrm{~m}$ de largo, en la que se marcaron tres bloques, distribuidos a lo largo de una transecta diagonal al terreno. Dentro de cada bloque ( $3 \mathrm{~m}$ x $3 \mathrm{~m}$ ) se tomaron muestras de suelo en forma sistemática, con arreglo aleatorio; las mismas correspondieron a tres posiciones equidistantes a lo largo de los surcos existentes y 
cuatro en los entresurcos. En cada uno de esos sitios se colectaron muestras a $7-15 \mathrm{~cm}$ y $22-30 \mathrm{~cm}$ de profundidad, coincidiendo con los horizontes $\mathrm{A}$ y $\mathrm{AB} / \mathrm{BA}$ respectivamente (Figura 1). Se obtuvo un total de 72 muestras.

A su vez, en la parcela muestreada se llevó a cabo un relevamiento planialtimétrico con cinta y nivel automático marca Pentax, Modelo AL 270, para determinar las direcciones del escurrimiento.

Con la finalidad de determinar humedad equivalente y densidad aparente se tomaron muestras de suelo en los mismos bloques considerados para la determinación de la presencia del patógeno. Se empleó un cilindro (alt.: $6 \mathrm{~cm}$ y diám.: 7,3cm), en 3 posiciones al azar y a profundidades equivalentes a los muestreos previamente descriptos.

Una vez llevadas las muestras al laboratorio, la presencia del patógeno se estableció en base a los métodos de Banihashemi \& Mitchell (2) y Jeffers \& Alwinckle (16). Cada muestra de suelo se homogeneizó, se dejó secar al aire y se desintegró empleándose un mortero. Posteriormente se extrajeron 2 submuestras de $10 \mathrm{~g}$ de suelo, las que se colocaron en placas de Petri sin medio. Las mismas se dejaron descubiertas y se expusieron a la luz natural durante 4 días a temperatura ambiente. Este nuevo período de deshidratación aseguró la muerte de todo resto vegetativo del pseudohongo y la luz indujo la germinación de las oosporas presentes (2).

Luego se aplicó un riego superficial, empleándose $6 \mathrm{~mL}$ de agua corriente por placa, incubándose $48 \mathrm{~h}$ bajo las condiciones previas. A continuación se agregaron $10 \mathrm{~mL}$ de agua corriente, quedando inundado el suelo e incubándose otras $48 \mathrm{~h}$ bajo las condiciones mencionadas. $\mathrm{La}$ formación de zoosporas se indujo volcando el excedente de agua y agregando $10 \mathrm{~mL}$ de agua corriente refrigerada a $8{ }^{\circ} \mathrm{C}$. Esa misma temperatura se mantuvo durante $2 \mathrm{~h}$ de incubación en oscuridad. Inmediatamente se colocaron cebos, los que fueron 6 cotiledones fotosintetizantes de manzana por placa, incubándose nuevamente a temperatura ambiente y bajo luz natural.

Una primera observación se realizó a las 48 h y una segunda al día siguiente. La presencia de cotiledones colonizados se estableció con microscopio estereoscópico y óptico en base a la presencia de zoosporangios característicos de $P$. cactorum creciendo en los márgenes de los cebos. Se consideró resultado positivo cuando hubo al menos un cotiledón por placa con presencia de $P$. cactorum.

En los casos en los que la morfología de los zoosporangios presentes no permitió identificar a la especie, se realizaron aislamientos. Para ello se colocaron los cotiledones infectados en heridas practicadas en manzanas previamente desinfectadas e incubándose a temperatura ambiente y oscuridad durante 5 días. Posteriormente se extrajeron porciones marginales de la pulpa colonizada de manzana, las que se sembraron en placas de Petri con agar V8; se incubó a $25-28{ }^{\circ} \mathrm{C}$, con un fotoperíodo de 12 horas de luz blanca y 12 horas de oscuridad, durante 8-10 días, hasta que desarrollaron estructuras reproductivas típicas de Phytophthora.

Se realizaron las determinaciones de los parámetros físicos del suelo de interés con las muestras extraídas con los cilindros. La densidad aparente (Dap) se calculó según:

Dap $=$ Peso del suelo seco/Vol. cilindro x 100 (20)

La humedad equivalente se determinó por el método de goteo (20). El mismo consistió en: $1^{\circ}$ ) en un vaso de precipitados de $250 \mathrm{~mL}$, se colocó un muestra de suelo, previamente pasada por un tamiz de $2 \mathrm{~mm}$, hasta completar las tres cuartas partes del volumen del recipiente; $2^{\circ}$ ) se apelmazó suavemente el contenido del vaso y luego se dejó gotear sobre el suelo $5 \mathrm{~mL}$ de agua; $3^{\circ}$ ) se dejó el vaso en reposo durante $20 \mathrm{~min}$., con la superficie tapada con papel humedecido. $4^{\circ}$ ) Luego se extrajo la fracción íntegramente humedecida, determinándose su peso húmedo y peso seco.

La humedad equivalente se calculó según:

Heq $=$ Peso del suelo húmedo - Peso del suelo seco/Peso del suelo seco x $100(20)$

Con los valores de densidad aparente y humedad equivalente se determinó la porosidad total del suelo y la macroporosidad para cada bloque. La porosidad total (Pt) se calculó según:

$\mathrm{Pt}=$ Dap/Densidad real $\mathrm{x} 100$

Densidad real: $2,55 \mathrm{gr} / \mathrm{cm}^{3}(20)$

\section{Horizonte A y AB/BA:}

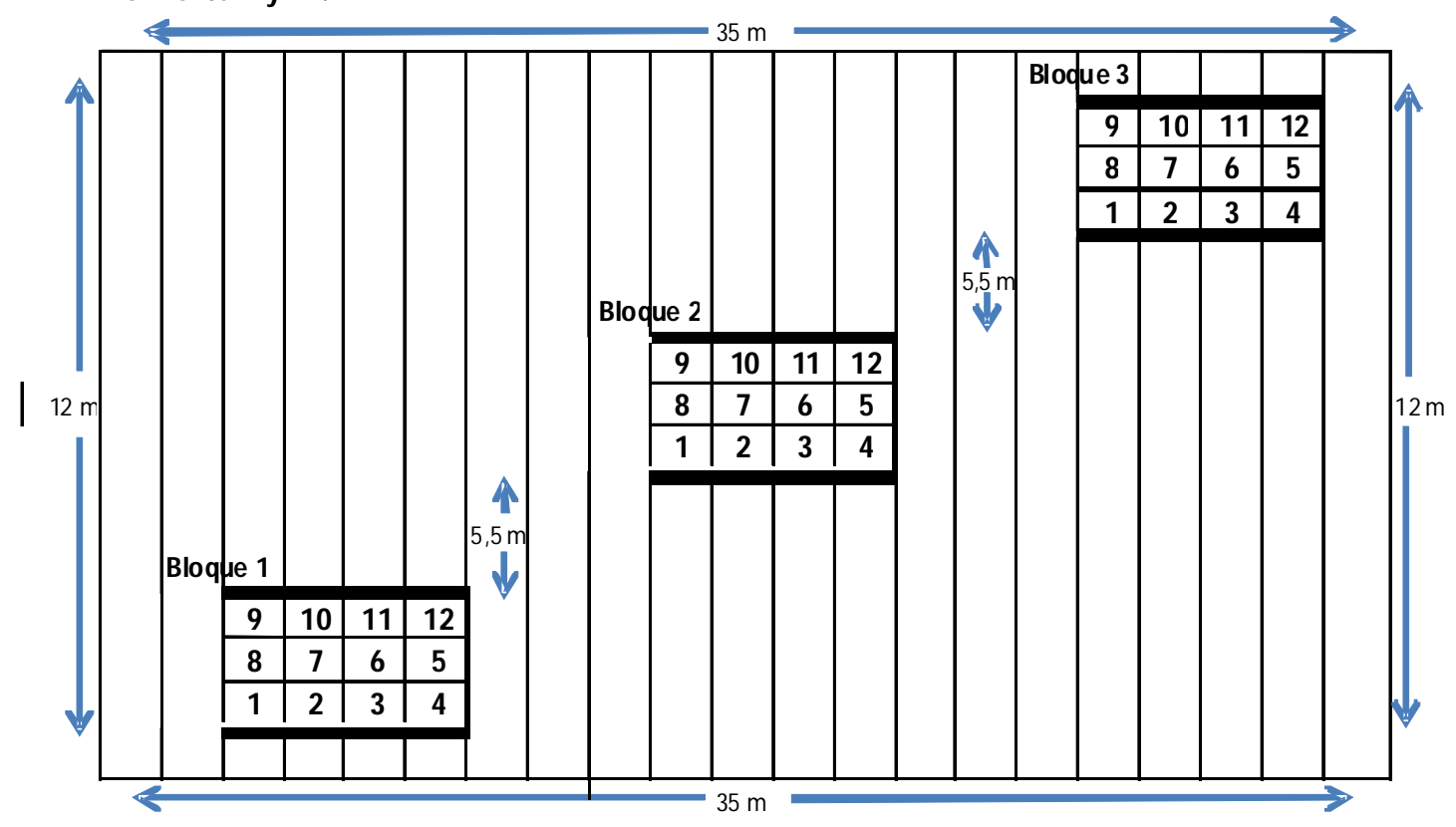

Figura 1. Relevamiento de datos de campo. Posición en el terreno. 
La Macroporosidad (Pa) se calculó según:

$\mathrm{Pa}=\mathrm{Pt}-\mathrm{HV}$ (Humedad volumétrica) $\quad \mathrm{HV}=$ Heq $\mathrm{x}$ Dap (20)

El análisis estadístico de los datos se realizó con el programa SPSS 11.5. Dado que el estudio de presencia de $P$. cactorum produjo una variable dicotómica, se aplicó la transformación Logit. El análisis de los datos se realizó mediante un modelo múltiple de Regresión Logística, para los factores posición dentro de los bloques y profundidad en el perfil de suelo (18).

El modelo aplicado fue:

$$
\log (p)=\ln \left(\frac{p}{1-p}\right)=\beta_{0}+\beta_{1} X_{1}+\beta_{2} X_{2}+\beta_{3} X_{3}+\varepsilon=\sum_{k=0}^{k 3} \beta_{k} X_{k}
$$

Donde:

$\mathrm{X}_{1=}$ posición transversal a los surcos

$\mathrm{X}_{2}$ = posición en entresurcos

$\mathrm{X}_{3=}$ profundidad

$\beta_{0:}$ constante

$\beta_{1} \beta_{2,} \beta_{3}$ : valores que permiten abordar la relación de dependencia entre la variable dependiente y la variable independiente o categórica.

\section{$\varepsilon=$ error aleatorio}

Para caracterizar los parámetros físicos del suelo: humedad equivalente y densidad aparente se utilizó un Análisis de Varianza con un Diseño en Bloques Completos Aleatorizados.

\section{RESULTADOS Y DISCUSIÓN}

La topografía de la parcela no mostró una pendiente definida en la extensión del lote, sino zonas de estancamiento y eventualmente rebalse, ante excesos de agua durante períodos de intensa precipitación (Figura 2).

Los cebos con presencia de $P$. cactorum estuvieron podridos y rodeados total o parcialmente por densas masas de zoosporangióforos cortos y compactos, con distribución simpodial de zoosporangios. Estos fueron papilados y ovoides, con un elevado nivel de homogeneidad en este último carácter así como en el tamaño.

Estas mismas características se observaron en las placas de Petri en las que se realizaron aislamientos; en las mismas también se formaron oogonios y anteridios con configuración parágina.
En base a dichas características se detectó $P$. cactorum en un máximo de 11 muestras superficiales en el bloque 1 y 8 muestras profundas en el bloque 3 (Tabla 1.a y Tabla 1.b), pero no se produjeron diferencias significativas tanto para los factores posición dentro de cada bloque y profundidad en el perfil del suelo como entre bloques. El factor que mostró una tendencia fue la profundidad, con un nivel de significancia de 0,089 $(\mathrm{p}<0,10)$. En dicho caso el coeficiente $\beta$ de la Regresión Logística arrojó un valor negativo $(\beta=-2,468)$, por lo que se asume que el efecto de la profundidad sobre la variable presencia es inverso (Tabla 2). Exploratoriamente podemos afirmar, dada la razón de probabilidades (Exp. $\beta<1$ ), que la profundidad podría comportarse como un factor de protección. Es decir, se observará una menor presencia de la enfermedad a mayor profundidad.

Los parámetros físicos del suelo, densidad aparente y humedad equivalente tuvieron valores medios de $1,33 \mathrm{gr} / \mathrm{cm}^{3}$ y $1,37 \mathrm{gr} / \mathrm{cm}^{3}$, y $21 \%$ y $16 \%$ para la profundidad 1 y 2 respectivamente. A su vez, no se observaron diferencias significativas para densidad aparente y humedad equivalente, a las profundidades consideradas $\left(\mathrm{p}_{\text {Dap }}=0,399 \mathrm{y}\right.$ $\left.\mathrm{p}_{\text {неq }}=0,201\right)$, así como tampoco entre bloques. La macroporosidad fluctuó entre 26 y 27 para la profundidad 1, y entre 24 y 25 para la profundidad 2.

El suelo del lote estudiado es representativo de la zona NE de la provincia de Bs. As. (28), así como la modalidad de los cultivos.

La falta de pendiente encontrada en el lote estudiado permitió la acumulación de agua en los entresurcos del cultivo de frutillas existente previamente al muestreo; este efecto resultó exacerbado dadas las características del lote, con similares valores de densidad aparente y humedad equivalente, que en conjunto indicarían un pobre nivel de macroporos en el suelo.

Reynolds et al. (23) demostraron que durante los episodios de lluvia, se produce una suspensión de zoosporangios y zoosporas de $P$. cactorum en el agua presente en los entresurcos, desde donde este inóculo resulta dispersado en forma ascendente por el impacto de las gotas de lluvia. La altura de los camellones más el canopeo de la frutillas constituyen, sin embargo, una barrera eficiente para la distribución lateral de ese inóculo $(11,19)$. Las oosporas también pueden ser desplazadas del mismo modo, una vez que se liberaron del interior de los tejidos en los que se formaron (14).

Cualquiera haya sido el mecanismo predominante, el método de detección empleado asegura, mediante la deshidratación de las muestras

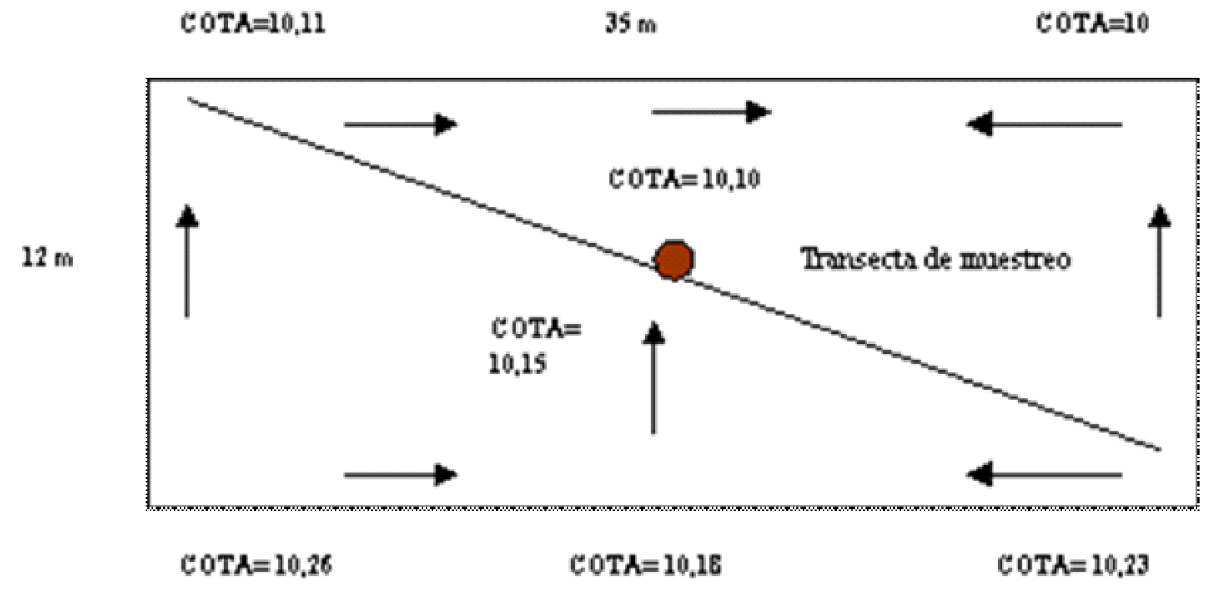

Figura 2. Relevamiento planialtimétrico de la parcela muestreada. 
Horizonte A:

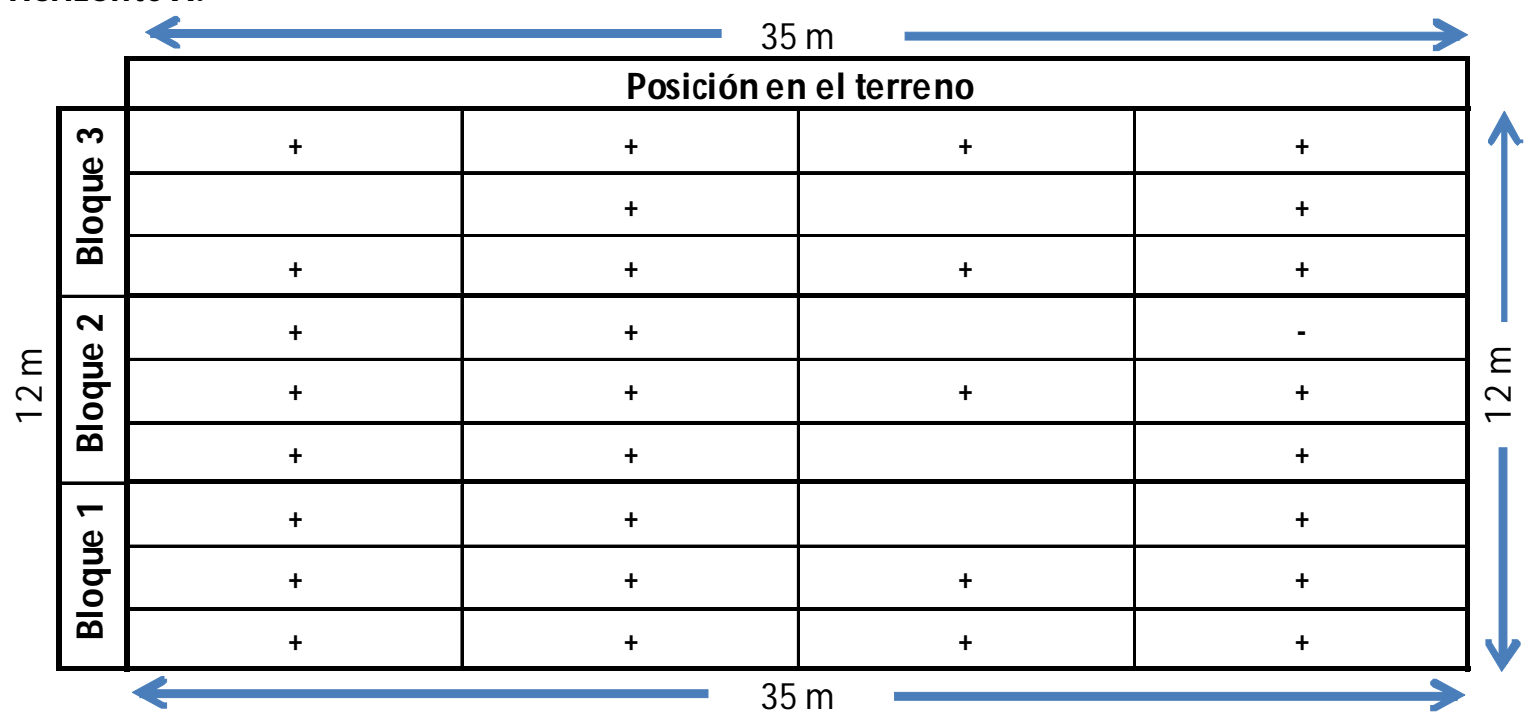

Tabla 1.a. Presencia/ausencia (+/-) de P. cactorum en el horizonte A para cada posición en el terreno.

\section{Horizonte A:}

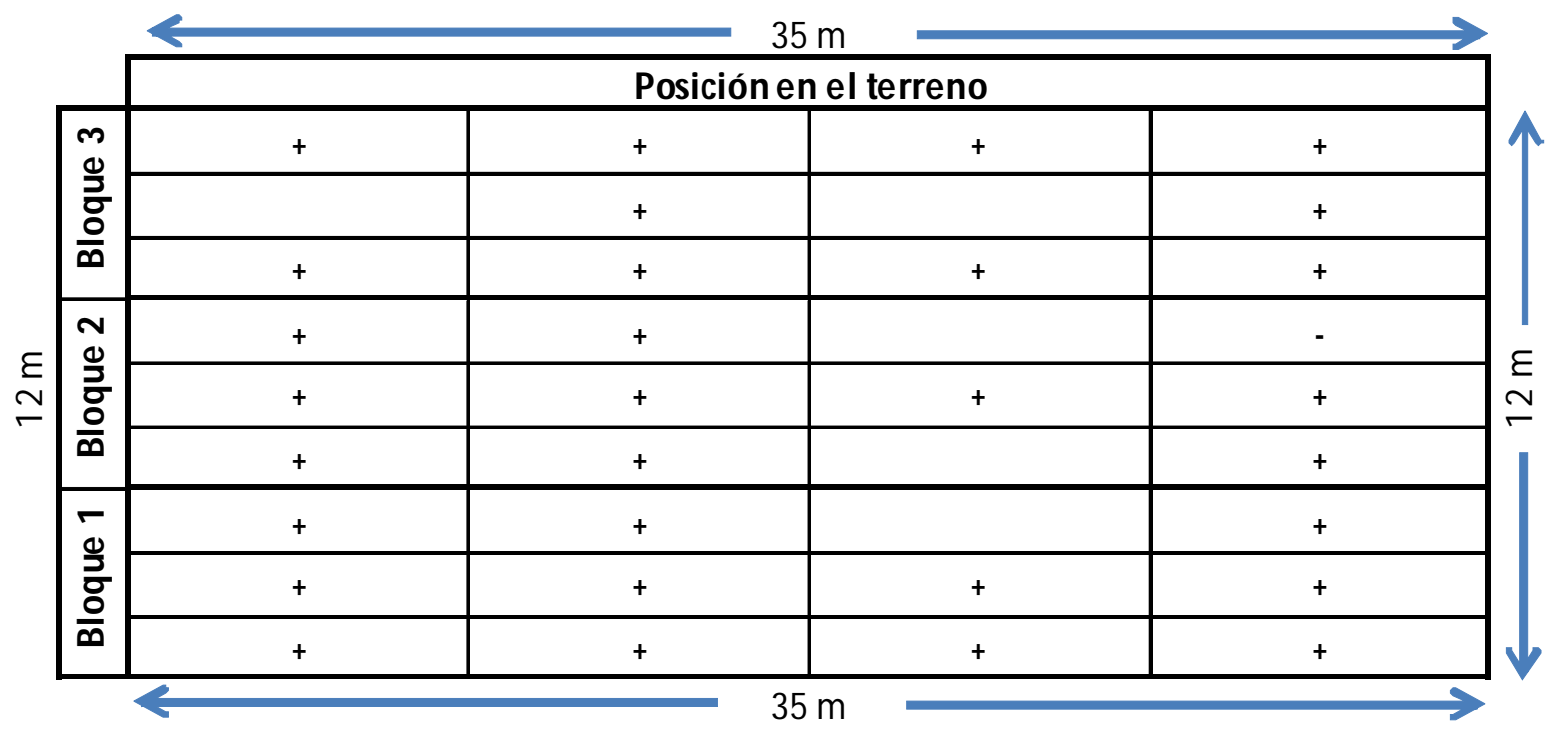

Tabla 1.b. Presencia/ausencia (+/-) de P. cactorum en el horizonte AB/BA para cada posición en el terreno.

de suelo, la muerte de toda estructura vegetativa (13), por lo que se evaluaron con exclusividad las poblaciones de oosporas.

Dicho método tuvo otras cualidades de interés. En primer lugar permitió comprobar que las oosporas, además de encontrarse presentes, produjeron finalmente zoosporas capaces de nadar hasta los cebos y transformarse en quistes infectivos; de este modo se demuestra al mismo tiempo presencia y patogenicidad (16). Por otro lado el método es simple, por lo que difícilmente se puedan introducir variaciones derivadas de la destreza de quien lo ejecuta, como resulta frecuente en métodos complejos. La única habilidad importante para llevar a buen término la aplicación de este método, es el reconocimiento de $P$. cactorum; ello es simple porque los zoosporangios presentan una morfología muy típica y porque los cebos empleados tienen un elevado nivel de especificidad para $P$. cactorum. Se debe destacar que la incubación bajo luz natural favorece la activación de las oosporas e induce a los zoosporangios a formar zoosporas. Se demostró así, que las oosporas con capacidad infectiva se encontraron presentes en proporciones elevadas, ya que de un total de 12 muestras por bloque y profundidad de muestreo, el menor valor correspondió al horizonte AB/BA, con 8 muestras con presencia de $P$. cactorum. También se comprobó que el patógeno presentó una distribución homogénea dentro de la parcela considerada, ya que no se encontraron diferencias significativas entre bloques. Se deberá tener en cuenta, sin embargo, que la escala de análisis determina en gran medida la variabilidad observada en cualquier sistema, por lo que el método de muestreo puede definir frecuentemente el patrón de distribución detectado (22).

Cuando se realizan muestreos de suelo para detectar diferentes especies de Phytophthora, típicamente se desprecian los primeros centímetros, ya que en ellos los extremos térmicos pueden afectar negativamente la supervivencia de los propágulos presentes (8). Eliminada esa capa, la distribución de las oosporas de $P$. cactorum en el perfil del suelo fue prácticamente homogénea, con tan sólo una pequeña tendencia hacia una disminución poblacional en profundidad. 


\begin{tabular}{|c|c|c|c|c|c|c|c|c|}
\hline & $\bar{\beta}$ & E.T. & Wald & $\begin{array}{l}\mathbf{g l} \\
\end{array}$ & Sig. & 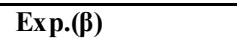 & \multicolumn{2}{|c|}{ I.C. 95\% para Exp.(ß) } \\
\hline $\begin{array}{l}\text { Variables en } \\
\text { la ecuación }\end{array}$ & & & & & & & Inferior & Superior \\
\hline POSFILA Paso 1(a) & & & $\mathbf{0 , 9 3 7}$ & 8 & 0,999 & & & \\
\hline POSFILA (1) & 20,041 & 13669,287 & $\mathbf{0 , 0 0 0}$ & 1 & 0,999 & 505709414,665 & 0,000 & . \\
\hline POSFILA (2) & 0,971 & 2,047 & 0,225 & 1 & 0,635 & 2,640 & 0,048 & 146,016 \\
\hline POSFILA (3) & 20,082 & 15685,877 & $\mathbf{0 , 0 0 0}$ & 1 & 0,999 & $\mathbf{5 2 6 5 3 2 4 9 5 , 5 0 0}$ & 0,000 & . \\
\hline POSFILA (4) & 20,448 & 12994,103 & $\mathbf{0 , 0 0 0}$ & 1 & 0,999 & 759011796,801 & 0,000 & . \\
\hline POSFILA (5) & 0,971 & 2,047 & 0,225 & 1 & 0,635 & 2,640 & 0,048 & 146,016 \\
\hline POSFILA (6) & $-0,515$ & 1,928 & $\mathbf{0 , 0 7 1}$ & 1 & $\mathbf{0 , 7 8 9}$ & 0,598 & 0,014 & 26,143 \\
\hline POSFILA (7) & 20,143 & 14940,942 & $\mathbf{0 , 0 0 0}$ & 1 & 0,999 & 559619287,642 & $\mathbf{0 , 0 0 0}$ & . \\
\hline POSFILA (8) & 19,871 & 12551,063 & $\mathbf{0 , 0 0 0}$ & 1 & 0,999 & 426354010,546 & 0,000 & . \\
\hline POSCOLUMNA & & & $\mathbf{0 , 0 4 3}$ & 3 & 0,998 & & & \\
\hline POSCOLUMNA(1) & 19,793 & 8454,812 & $\mathbf{0 , 0 0 0}$ & 1 & 0,998 & 394498010,952 & 0,000 & . \\
\hline POSCOLUMNA(2) & 20,550 & 9877,987 & 0,000 & 1 & 0,998 & 840600025,936 & 0,000 & - \\
\hline POSCOLUMNA(3) & 0,275 & 1,334 & 0,043 & 1 & $\mathbf{0 , 8 3 7}$ & 1,317 & 0,096 & 17,996 \\
\hline PROFUNDIDAD(1) & $-2,468$ & 1,449 & 2,900 & 1 & 0,089 & 0,085 & 0,005 & 1,451 \\
\hline Constante & 1,611 & 1,651 & $\mathbf{0 , 9 5 2}$ & 1 & 0,329 & 5,010 & & \\
\hline
\end{tabular}

Tabla 2. Regresión logística

Donde:

$\beta$ : Coeficiente de Regresión logística, E.T.: Error típico, Wald: Estadística de prueba, gl.: Grados de libertad, Sig.: Nivel de significancia o valor p, Exp. ( $\beta$ ): Razón de probabilidades (odds), I.C.: Intervalo de confianza.

Se demostró de este modo que el laboreo del suelo distribuye a las oosporas de dicho patógeno en profundidad y en cantidades importantes, a pesar de que la mayor proporción de las mismas se produce en superficie, donde quedan expuestas a condiciones adversas, por lo que podrían perecer. También la falta de diferencias en profundidad de los parámetros físicos del suelo considerados es un indicador de la inversión de los horizontes producida por el arado.

Los resultados obtenidos indican que bajo las condiciones de un lote como el estudiado se deberá asegurar que los fumigantes aplicados penetren profundamente en el suelo y difundan correctamente en todo el perfil.

La solarización puede resultar inefectiva porque el incremento de temperatura que es posible lograr en profundidad puede ser insuficiente para afectar la viabilidad de las oosporas de $P$. cactorum, especialmente si el suelo se encuentra seco $(2,16)$.

Otra posibilidad de manejo en suelos con cultivos de frutillas con presencia de $P$. cactorum, podría ser la siembra directa, pero esta es una práctica que por ahora no se aplica en los cultivos hortícolas de la zona.

Por otro lado, la rotación de cultivos sería efectiva para este patosistema, considerando la alta especificidad de los aislamientos de $P$. cactorum que afectan a las frutillas. En tal caso se debe asegurar la desaparición del inóculo existente, para lo cual se debería evaluar experimentalmente el tiempo necesario para que ello ocurra.

En términos generales es apropiado afirmar, como lo hicieron Campbell y Neher (3) que "una adecuada planificación de las tácticas de control de los patógenos del suelo y de las enfermedades que causan, se fundamenta en un conocimiento profundo de la distribución espaciotemporal de esos microorganismos, así como de las interacciones que se producen".

\section{REFERENCIAS}

1. Brasier, C. M.; Hansen, E. M. Evolutionary biology of Phytophthora. Annual Review of Phytopathology, Palo Alto, v. 30, 
p. 173-200, 1992

2. Banihashemi, Z.; Mitchell, J. E. Factors affecting oospore germination in Phytophthora cactorum, the incitant of apple collar rot. Phytopathology, St. Paul, v. 66, p. 443-448, 1976.

3. Campbell, C. L.; Neher, D. A. Challenges, opportunities, and obligations in root disease epidemiology and management. In: Hall, R. Principles and practice of managing soilborne plant pathogens. St. Paul: APS, 1993. p. 20-49.

4. Duniway, J. M. Movement of zoospores of Phytophthora cryptogea in soils of various textures and matrix potentials. Phytopathology, St. Paul, v. 66, p. 877-882, 1976.

5. Duniway, J. M. Role of physical factors in the development of Phytophthora diseases. In: Erwin, D. C.; Bartnicki-Garcia, S.; Tsao, P. H. Phytophthora: Its biology, taxonomy, ecology and pathology. St. Paul: APS, 1983. p. 175-188.

6. Eikemo, H.; Klemsdal, S. S.; Riisberg, I.; Bonants, P.; Stensvand, A.; Tronsmo, A. Genetic variation between Phytophthora cactorum isolates differing in their ability to cause crown rot in strawberry. Mycological Research, London, v.108, p. 317-324, 2004.

7. Ellis M. A.; Madden, L. V. Leather rot. In: Maas J. L. Compendium of strawberry diseases. 2 ed. St. Paul: APS, 1998. p. 33-35.

8. Erwin, D. C.; Ribero O. K. (Ed.) Phytophthora Diseases Worldwide. St. Paul: The American Phytopathological Society, 1996. $562 \mathrm{p}$.

9. González, B. A.; Steciow, M. M.; Arambarri, A. M. Presencia de Phytophthora cactorum en lotes de producción comercial de frutillas de la zona norte de la provincia de Buenos Aires. Boletín de la Sociedad Argentina de Botánica, Buenos Aires, v. 38, p. 264-265, 2003.

10. Grove, G. G.; Ellis, M. A.; Madden, L. V.; Schmitthenner, A. F. Overwinter survival of Phytophthora cactorum in infected strawberry fruit. Plant Diseases, St. Paul, v. 69, p. 514-515, 1985.

11. Grove, G. G.; Madden, L. V.; Ellis, M. A. Splash dispersal of Phytophthora cactorum from infected strawberry fruit. Phytopathology, St. Paul, v. 75, p. 611-615, 1985.

12. Hantula, J.; Lilja, A.; Nuorteva, H.; Parikka, P.; Werres, S. Pathogenicity, morphology and genetic variation of Phytophthora cactorum from strawberry, apple, rhododendron, and silver bich. Mycological Research, London, v. 104, p. 1062-1068, 2000.

13. Horner. I. J.; Wilcox, W. F. Spatial distribution of Phytophthora cactorum in New York apple orchard soils. Phytopathology, St. Paul, v. 86, p. 1122-1131, 1996.

14. Horner, I. J.; Wilcox, W. F. Temporal changes in activity and dormant spores populations of Phytophthora cactorum in New York apple orchards soils. Phytopathology, St. Paul, v. 86, p. 1133-1139, 1996 .

15. Huang, H; Jeffers, S. N.; Layne, D. R.; Schnabel, G. AFLP Analysis of Phytophthora cactorum isolates from strawberry and other hosts: Implications for identifying the primary source of inoculum. Plant Diseases, St. Paul, v. 88, p. 714-720, 2004

16. Jeffers, S. N.; Aldwinckle, H. S. Enhancing detection of Phytophthora cactorum in naturally infected soil. Phytopathology, St. Paul, v. 77, p. 1475-1482, 1987.

17. Juarez-Palacios, C.; Felix-Gastelum, R.; Wakeman, R. J.; Paplomatas, E. J.; DeVay, J. E. Thermal sensitivity of three species of Phytophthora and the effect of soil solarization on their survival. Plant Diseases, St. Paul, v. 75, p. 1160-1164, 1991.

18. Little, T. M.; Hills, J. F. Métodos estadísticos para la investigación en la agricultura. 2 ed. México D. F.: Trillas, 1991. $270 \mathrm{p}$.

19. Madden, L. V.; Ellis, M. A.; Grove, G. G.; Reynols, K. M.; Wilson, L. L. Epidemiology and control of leather rot of strawberries. Plant Diseases, St. Paul, v. 75, p. 439-446, 1991.

20. Mizuno, I.; Arrigo, N. M. Guía de trabajos prácticos de edafología. Buenos Aires: FAUBA. 1988. 25p.

21. Nicot, P. C.; Rouse, D. I.; Vandell, B. S. Comparison of statistical methods for studying spatial patterns of soilborne plant pathogens in the field. Phytopathology, St. Paul, v. 74, p. 1399-1402, 1984.

22. Pryce, J.; Edwards, W.; Gadek, P. Distribution of Phytophthora cinnamomi at different spatial scales: When can a negative result be considered positively?. Austral Ecology, Carlton, v. 27, p. 459-462, 2002.

23. Reynolds, K. M.; Madden, L. V.; Reichard, D. L.; Ellis, M. A Splash dispersal of Phytophthora cactorum from infected strawberry fruit by simulated canopy drip. Phytopathology, St. Paul, v. 79, p. 425-432, 1989.

24. Ristaino, J. B.; Gumpertz, M. New frontiers in the study of dispersal and spatial analysis of epidemics caused by species in the genus Phytophthora. Annual Revision of Phytopathology, North Carolina, v. 38, p. 541-576, 2000.

25. Seemüller, E. Crown rot. In: Mass, J. L. Compendium of strawberry diseases. 2 ed. St. Paul: APS, 1998. p. 50-51.

26. Sneh, B.; McIntosh, D. L. Studies of the behaviour and survival of Phytophthora cactorum in soil. Canadian Journal of Botany, Young House, v. 52, p. 795-802, 1974.

27. Workneh, F.; Yang, X. B.; Tylka, G. L. Effect of tillage practices on vertical distribution of Phytophthora sojae. Plant Diseases, St. Paul, v. 82, p. 1258-1263, 1998.

28. Atlas de Buenos Aires. Disponible en: <http:// www.atlasdebuenosaires.gov.ar>. Acceso en: 16 junio 2007.

29. Argentina. Servicio Meteorológico Nacional (SMN). Disponible en: <http://www.smn.gov.ar>. Acceso en: 16 junio 2007.

30. Argentina. Secretaría de Agricultura Ganadería y Pesca de La Nación. Disponible en: <http://www.sagpya.mecom.gov.ar>. Acceso en: 20 junio 2007. 